WS15-B01

\title{
Distributed Acoustic Sensing for Borehole Seismic Applications
}

\author{
T.R. Parker* (Silixa Ltd)
}

\section{SUMMARY}

Distributed acoustic sensing (DAS) technology enables the simultaneous collection of acoustic data at all points along a length of optical fibre. In this presentation, we look at the main options for the installation geometry (the cable deployed in the production tubing, clamped to the outside of the production tubing or cemented behind the casing) and discuss the effect of this geometry on the measured DAS signal. This comparison will examine the signal coupling from the formation to the fibre, and the resultant influence on the signal to noise ratio, and also look at the relative strength of the measured flow signal, in the case of a flowing well. Through this discussion, we will explain the important trade-off between the chosen DAS spatial resolution and the resultant signal-to-noise ratio.

We will then extend the discussion to look at ways to tune the directionality of the DAS measurement and to tailor the DAS response to resemble the output from different standard geophysical sensor families. Through this presentation, we will aim to demonstrate the flexibility of the DAS sensing mechanism, and explain how this flexibility enables the dynamic optimisation of the system performance for various target measurements. 


\section{Amsterdam ' 14}

Distributed acoustic sensing (DAS) technology enables the simultaneous collection of acoustic data at all points along a length of optical fibre. Over the past few years, a large number of surface and borehole DAS surveys have been conducted successfully, producing a rich and comprehensive data set with minimal disturbance to normal oilfield operation.

Physically, in the case of the particular DAS architecture discussed in this presentation, the DAS measures the average axial strain rate at discrete intervals (typically $1 \mathrm{~m}$ to $10 \mathrm{~m}$ ) all along the sensing fibre. This fibre is housed in a protective cable which, in the case of VSP surveys, is installed in the borehole in one of a number of possible configurations. In this presentation, we look at the main options for the installation geometry (the cable deployed in the production tubing, clamped to the outside of the production tubing or cemented behind the casing) and discuss the effect of this geometry on the measured DAS signal. This comparison will examine the signal coupling from the formation to the fibre, and the resultant influence on the signal to noise ratio, and also look at the relative strength of the measured flow signal, in the case of a flowing well. Through this discussion, we will explain the important trade-off between the chosen DAS spatial resolution and the resultant signal-to-noise ratio.

We will then extend the discussion to look at ways to tune the directionality of the DAS measurement and to tailor the DAS response to resemble the output from different standard geophysical sensor families. Through this presentation, we will aim to demonstrate the flexibility of the DAS sensing mechanism, and explain how this flexibility enables the dynamic optimisation of the system performance for various target measurements. 